\title{
The homology of the Milnor fiber for classical braid groups
}

\author{
FilipPo CALlegaro
}

\begin{abstract}
In this paper we compute the homology of the braid groups, with coefficients in the module $\mathbb{Z}\left[q^{ \pm 1}\right]$ given by the ring of Laurent polynomials with integer coefficients and where the action of the braid group is defined by mapping each generator of the standard presentation to multiplication by $-q$.

The homology thus computed is isomorphic to the homology with constant coefficients of the Milnor fiber of the discriminantal singularity.
\end{abstract}

20F36; 20J06, 32S55

\section{Introduction}

Let $(W, S)$ be a Coxeter system, with $W$ a finite, irreducible Coxeter group and let $G_{W}$ be the associated Artin group (see Bourbaki [5] for an introduction to Coxeter groups and their classifications and Brieskorn and Saito [6] for relations between Coxeter groups and Artin groups ). The main objects of study of this paper are the Artin groups of type $\mathrm{A}_{n}$. We recall that the Artin group $G_{\mathrm{A}_{n}}$ is the same as the classical braid group (see Artin [3] for the original definition and Birman [4] for more details) on $n+1$ strands $\operatorname{Br}(n+1)$ and a standard presentation is given by:

$$
\left.\left\langle\sigma_{i}, i=1, \ldots, n\right| \sigma_{i} \sigma_{i+1} \sigma_{i}=\sigma_{i+1} \sigma_{i} \sigma_{i+1}, \sigma_{i} \sigma_{j}=\sigma_{j} \sigma_{i} \text { if }|i-j| \geq 2\right\rangle .
$$

The homology and cohomology with trivial coefficients for Artin groups $G_{W}$ associated to Coxeter groups $W$ of finite type are well known. The first partial computations for the cohomology of braid groups are due to Arnol'd and appear in [1] and [2]. In [17] Fuks computes the cohomology ring of braid groups with $\mathbb{Z}_{2}$-coefficients using a cell decomposition of the Alexandroff compactification of the configuration spaces of $\mathbb{C}$, which are classifying spaces for braid groups. Using the same cell decomposition, in [24] Vaŭnšteřn computes the cohomology ring of braids with $\mathbb{Z}_{p}-$ coefficients for any prime $p$; moreover he computes the Bockstein operator, hence he gets the cohomology ring of braids with $\mathbb{Z}$-coefficients. The same results in homology are obtained independently by F Cohen in [12]: he computes the homology of braid groups over $\mathbb{Z}$ using the theory of homology operations in $n$-fold loop spaces. In [19] Gorjunov extends the results of Vaŭnšteĭn computing the cohomology ring of Artin 
groups of type $\mathrm{C}_{n}$ and $\mathrm{D}_{n}$ over $\mathbb{Z}$. Finally in [23] Salvetti computes the cohomology groups with $\mathbb{Z}$-coefficients for the Artin groups associated with exceptional Coxeter groups; the ring structure is determined by Landi in [21].

Let $\mathbf{X}_{W}$ be the classifying space for $G_{W}$. For a ring $A$ we can define the local system $A\left[q^{ \pm 1}\right]$ over the space $\mathbf{X}_{W}$, with twisted coefficients over the ring $A\left[q^{ \pm 1}\right]$, where each standard generator of $G_{W}$ acts as multiplication by $-q$.

The homology groups $H_{*}\left(\mathbf{X}_{W} ; A\left[q^{ \pm 1}\right]\right)$ are equal to the homology group (with trivial coefficients over the ring $A$ ) of the Milnor fiber $\mathbf{F}_{W}$ of the discriminant singularity associated to $W$. The same hold for the cohomology groups, modulo an index shifting ((as proved by the author in [7]). Hence we get a $q$-module structure on the homology and cohomology of the Milnor fiber $\mathbf{F}_{W}$, where the $-q$-multiplication corresponds to the map induced by the monodromy automorphism of the fiber. We remark also that for the case $\mathrm{A}_{n}$ the fiber $\mathbf{F}_{\mathrm{A}_{n}}$ is a classifying space for the commutator subgroup of the braid group $\operatorname{Br}(n+1)$, hence the homology (cohomology) of the fiber is also the homology (cohomology) of $\operatorname{Br}(n+1)^{\prime}$.

The groups $H^{*}\left(\mathbf{X}_{W} ; A\left[q^{ \pm 1}\right]\right)$ have been computed by the author and Salvetti [9] for all exceptional Artin groups. Several authors (De Concini, Procesi and Salvetti [14], Frenkel' [16], Markaryan [22]) made this computation for type $\mathrm{A}_{n}$ Artin groups, with $A$ a field of characteristic 0. In de Concini, Procesi, Salvetti and Stumbo [15] the computations have been performed for all other finite type Artin groups, with rational coefficients. In all these cases all cohomology modules are sums of cyclotomic fields (or zero). In particular, in [14], an interesting arithmetic behaviour of the table $\left(H^{i}\left(\operatorname{Br}(j) ; A\left[q^{ \pm 1}\right]\right)\right)_{i, j}$ is noticed.

In [22] Markaryan used the isomorphism between the standard resolution of a certain algebra and the algebraic complex associated to the classifying spaces for braid groups to compute the homology of braid groups with coefficients in the local system $\mathbb{Q}\left[q^{ \pm 1}\right]$.

In this paper we extend the use of this resolution in order to compute the homology of braid groups with coefficients in the local system $K\left[q^{ \pm 1}\right]$ for a generic field $K$. We also compute the Bockstein operator in order to get the homology over $\mathbb{Z}\left[q^{ \pm 1}\right]$. Some computations for the first cases can be found in tables 1,2,3 and 4. Our main result is stated in Theorem 4.

In [13] Cohen and Pakianathan compute the homology of the braid group on infinitely many strands $\operatorname{Br}(\infty)$ with coefficients in the local system $K\left[q^{ \pm 1}\right]$ for any field $K$ : this is the stable part of the homology of $\operatorname{Br}(n)$ (with coefficients the same local system) with respect to the embeddings $j_{n}: \operatorname{Br}(n) \hookrightarrow \operatorname{Br}(n+1)$. In Corollary 5.1 we obtain the same result; moreover we are able to compute the Bockstein operator, hence we give a presentation of the homology of $\operatorname{Br}(\infty)$ with coefficients in the local system $\mathbb{Z}\left[q^{ \pm 1}\right]$. 
Corollary 5.1 We have that $H_{*}\left(\operatorname{Br}(\infty) ; \mathbb{Q}\left[q^{ \pm 1}\right]\right)=\mathbb{Q}$, concentrated in dimension 0 ;

$$
H_{*}\left(\operatorname{Br}(\infty) ; \mathbb{Z}_{2}\left[q^{ \pm 1}\right]\right)=\mathbb{Z}_{2}\left[x_{2}^{2}, x_{2^{i}}, i>1\right]
$$

and for a prime $p>2$

$$
H_{*}\left(\operatorname{Br}(\infty) ; \mathbb{Z}_{p}\left[q^{ \pm 1}\right]\right)=\mathbb{Z}_{p}\left[y_{2 p^{i}}, x_{2 p^{i}}, i>0\right] /\left(x_{2 p^{i}}^{2}\right)
$$

with $\operatorname{dim} x_{i}=i-1, \operatorname{dim} y_{i}=i-2$. The Bockstein operator acts as follows:

$$
\beta_{2} x_{2^{i}}=x_{2^{i-1}}^{2} ; \quad \beta_{p} y_{i}=0 ; \quad \beta_{p} x_{i}=y_{i} \quad(\text { for } p>2) .
$$

The homology $H_{*}\left(\operatorname{Br}(\infty) ; \mathbb{Z}\left[q^{ \pm 1}\right]\right)$ has no $p^{2}$-torsion for any prime $p$. A presentation of $H_{*}\left(\operatorname{Br}(\infty) ; \mathbb{Z}\left[q^{ \pm 1}\right]\right)$ is given by

$$
\mathbb{Z}\left[\begin{array}{c}
y_{2 p^{i}}, x_{2^{j}}^{2}, \\
x_{2^{i}}^{2} x_{2^{i_{1}}} \cdots x_{2^{i} h} \\
y_{2 p^{j}} x_{2 p^{j_{1}}} \cdots x_{2 p^{j_{h}}}
\end{array}\right] /\left(2 x_{2^{i}}, p y_{2 p^{j}}, x_{2 p^{j}}^{2}\right)
$$

with indices running as follows: $0<i, i+1<i_{1}<\cdots<i_{h}, 0<j<j_{1}<\cdots<j_{h}$ and $p$ in the set of odd primes. The structure of $\mathbb{Z}\left[q^{ \pm 1}\right]$-module is trivial and so the action of $q$ corresponds to multiplication by -1 .

\section{Notation and definitions}

Let $R$ be a ring with identity and let $q$ be an element of $R$. Following [22] we define the algebra of $q$-divided polynomials $\Gamma_{R}(t, q)$ as the graded algebra over $R$ with generators $t_{i}\left(i \in \mathbb{N}, \operatorname{deg} t_{i}=i\right)$ and relations

$$
t_{i} t_{j}=\left[\begin{array}{c}
i+j \\
i
\end{array}\right] t_{i+j}
$$

where $\quad\left[\begin{array}{c}i+j \\ i\end{array}\right]=\frac{(1-q)\left(1-q^{2}\right) \cdots\left(1-q^{i+j}\right)}{(1-q) \cdots\left(1-q^{i}\right)(1-q) \cdots\left(1-q^{j}\right)}$

is the $q$-binomial coefficient; if we define the $q$-analog of $i$ as

$$
[i]=1+q+\cdots+q^{i-1},
$$

and $[i] !=[2][3] \cdots[i]$, then we can write also

$$
\left[\begin{array}{c}
i+j \\
i
\end{array}\right]=\frac{[i+j] !}{[i] ![j] !}
$$

Algebraic $8 \mathcal{G}$ Geometric Topology, Volume 6 (2006) 
We recall that if $q$ commutes with $a$ and $b$ and $b a=q a b$, then

$$
(a+b)^{n}=\sum_{i=0}^{n}\left[\begin{array}{l}
n \\
i
\end{array}\right] a^{i} b^{n-i} .
$$

Now we want to study the homology and cohomology (as defined in Cartan and Eilenberg [10]) of the algebra $\Gamma_{R}(t, q)$. We can consider the normalized standard complex (see [10] for a general definition) that calculates the homology of the algebra $\Gamma_{R}(t, q)$. The complex is given as follows:

$$
0 \leftarrow R=C_{0} \stackrel{\partial}{\leftarrow} C_{1} \stackrel{\partial}{\leftarrow} C_{2} \stackrel{\partial}{\leftarrow} \cdots,
$$

where the $R$-module $C_{n}$ is freely generated by all the monomials of the form $a \otimes t_{i_{1}} \otimes$ $\cdots \otimes t_{i_{n}}$, with $a \in R$ and the boundary formula is:

$$
\begin{aligned}
& \partial\left(a \otimes t_{i_{1}} \otimes \cdots \otimes t_{i_{n}}\right)= \\
= & \sum_{j=1}^{n-1}(-1)^{j+1} a \otimes t_{i_{1}} \otimes \cdots \otimes t_{i_{j}} t_{i_{j+1}} \otimes \cdots \otimes t_{i_{n}}= \\
= & \sum_{j=1}^{n-1}(-1)^{j+1}\left[\begin{array}{c}
i_{j}+i_{j+1} \\
i_{j}
\end{array}\right] a \otimes t_{i_{1}} \otimes \cdots \otimes t_{i_{j}+i_{j+1}} \otimes \cdots \otimes t_{i_{n}} .
\end{aligned}
$$

By means of the grading of the algebra $\Gamma_{R}(t, q)$, the module $C$ is decomposed into the direct sum of complexes of different degrees,

$$
C=\bigoplus_{i=0}^{\infty} C^{(i)},
$$

where $\operatorname{deg}\left(a \otimes t_{i_{1}} \otimes \cdots \otimes t_{i_{n}}\right)=i_{1}+\cdots+i_{n}$ and for $c \in C_{k}^{(n)}$ we set $\operatorname{deg} c=n$ and $\operatorname{dim} c=k$.

The dual complex $C^{*}$, given by the modules $C^{n}=\operatorname{Hom}\left(C_{n}, R\right)$ and with coboundary map the transposed map of $\partial$, computes the cohomology ring of the algebra $\Gamma_{R}(t, q)$. The multiplication is defined on representatives as follows: if $m_{1}^{*}$ and $m_{2}^{*}$ are the dual classes of the monomials $m_{1}$ and $m_{2}$, respectively, then the product $m_{1}^{*} m_{2}^{*}$ is the dual class of the monomial $m_{1} \otimes m_{2}$.

Given a space $X$ such that $\pi_{1}(X)=\operatorname{Br}(n)$, we can define a local system $R$ on $X$. Over a point $x \in X$ we have the ring $R$; the system of coefficients is twisted and the action is given by sending each standard generator of the group $\operatorname{Br}(n)$ to multiplication by $-q$. This action corresponds to the determinant of the Burau representation for the braid group $\operatorname{Br}(n)$ (see, for example, [13]). We remark that although the choice of the 
multiplication by $q$ would be equivalent, we use $-q$, which seems more natural to us, and also for coherence with [13], [14], [15].

The complex $C_{n-*}^{(n)}$ coincides with the complex (defined in [23]) that computes the cohomology of the group $\operatorname{Br}(n)$ with local coefficients $R$.

By the module $H_{*}(\operatorname{Br}(*), R)$ we mean the bigraded module (the gradings are the degree deg and the dimension $\operatorname{dim}$ ) whose component of degree $n$ and dimension $l$ is $H_{l}(\operatorname{Br}(n), R)$. We can think of $H_{*}(\operatorname{Br}(*), R)$ as a ring using the multiplication induced by the standard homomorphism (obtained by juxtaposing braids)

$$
\mu_{i j}: \operatorname{Br}(i) \times \operatorname{Br}(j) \rightarrow \operatorname{Br}(i+j) .
$$

Theorem 1 [22] The ring $H_{*}(\operatorname{Br}(*), R)$ coincides, modulo a change of indexes, with the cohomology ring of the algebra $\Gamma_{R}(t, q)$ :

$$
H_{l}(\operatorname{Br}(n), R) \simeq H^{n-l}\left(\Gamma_{R}(t, q)\right)_{(\operatorname{deg}=n)}
$$

and the product structure in $H_{*}(\operatorname{Br}(*), R)$ coincides with the cohomological multiplication of the ring $H^{*}\left(\Gamma_{R}(t, q)\right)$.

\section{The Milnor fiber and some lemmas}

Let $V=\mathbb{C}^{n}$ be a finite-dimensional complex vector space. The symmetric group on $n$ elements $\mathfrak{S}_{n}$ acts on this space by permuting the coordinates. Let $l_{i j}$ be the linear functional $z_{i}-z_{j}$ and let $\mathcal{H}_{i j}$ be the hyperplane $\left\{l_{i j}=0\right\}$. The complement of the union of the hyperplanes

$$
\mathbf{Y}_{n}=V \backslash \bigcup_{i<j} \mathcal{H}_{i j}
$$

is a classifying space for the pure braid group on $n$ strands. If we consider the quotient of $\mathbf{Y}_{n}$ with respect to the action of $\mathfrak{S}_{n}$

$$
\mathbf{X}_{n}=\mathbf{Y}_{n} / \mathfrak{S}_{n}
$$

we get a classifying space for the braid group $\operatorname{Br}(n)$. Consider the product $\delta=\prod_{i<j} l_{i j}^{2}$. The polynomial $\delta$ is invariant with respect to the action of $\mathfrak{S}_{n}$ and so it induces a map

$$
\delta^{\prime}: \mathbf{X}_{n} \rightarrow \mathbb{C}^{*} .
$$

The fiber $\mathbf{F}_{1}(n)=\delta^{\prime-1}(1)$ is the Milnor fiber of the discriminant singularity $\mathbf{F}_{0}(n)=$ $\cup \mathcal{H}_{i j} / \mathfrak{S}_{n}$ in the affine variety $V / \mathfrak{S}_{n}$ (which is isomorphic to the complex space $\mathbb{C}^{n}$ ). The complement of $\mathbf{F}_{0}(n)$ in $V / \mathfrak{S}_{n}$ can also be thought as the set of polynomials with distinct roots in the space of all monic polynomials of degree $n$ with complex 
coefficients. Moreover, the fiber $\mathbf{F}_{1}(n)$ is a classifying space for the commutator subgroup $\operatorname{Br}(n)^{\prime}$ of the braid group $\operatorname{Br}(n)$ and we have that (see for example [7]):

$$
H_{*}\left(\operatorname{Br}(n)^{\prime}, \mathbb{Z}\right) \simeq H_{*}\left(\mathbf{F}_{1}(n), \mathbb{Z}\right) \simeq H_{*}\left(\operatorname{Br}(n), \mathbb{Z}\left[q^{ \pm 1}\right]\right)
$$

and

$$
H^{*}\left(\operatorname{Br}(n)^{\prime}, \mathbb{Z}\right) \simeq H^{*}\left(\mathbf{F}_{1}(n), \mathbb{Z}\right) \simeq H^{*+1}\left(\operatorname{Br}(n), \mathbb{Z}\left[q^{ \pm 1}\right]\right),
$$

with the usual $q$-action.

In what follows $K$ is a field and $p$ always refers to the characteristic of the field $K$ ( $p=0$ or $p$ a prime). Cyclotomic polynomials are usually defined over a field of characteristic 0 , by saying that the $m$-th cyclotomic polynomial is the monic polynomial whose roots are all simple roots and are all the $m$-th primitive roots of unity. Over a generic field $K$ we can define by induction the $m$-th cyclotomic polynomial $\varphi_{m}$, by saying that $\varphi_{1}=q-1$ and $q^{m}-1=\prod_{i \mid m} \varphi_{i}$. For each positive integer $m$ we define the ring $K(m)=K[q] / \varphi_{m}$.

We have the following technical lemmas:

Lemma 3.1 [8] Let $m<n$ be two positive integers. Then we have:

$$
\left(\varphi_{m}, \varphi_{n}\right)=\left\{\begin{array}{cl}
\left(\varphi_{m}, p\right) & \text { if } n=m p^{i}, i \geq 1, \text { for a prime } p \\
(1) & \text { otherwise. }
\end{array}\right.
$$

We leave the proof to the Appendix. As an easy consequence of this Lemma we obtain the following Corollary, whose proof is left to the reader:

Corollary 3.2 Let $i<j$ be two positive integers. Then we can write

$$
\varphi_{p^{j}}=\varphi_{p^{i}} \omega+p \psi
$$

where $\omega, \psi \in \mathbb{Z}\left[q^{ \pm 1}\right]$ and $\psi$ is invertible $\bmod \varphi_{p^{i}} ;$

$$
\varphi_{m p^{j}}=\varphi_{m p^{i}} \omega+p \psi
$$

where $\omega, \psi \in \mathbb{Z}\left[q^{ \pm 1}\right]$ and $\psi$ is invertible $\bmod \varphi_{m p^{i}}$.

We can fix once and for all polynomials $\omega_{p^{j}, p^{i}}, \omega_{m p^{j}, m p^{i}}, \psi_{p^{j}, p^{i}}, \psi_{m p^{j}, m p^{i}}$ satisfying the equations (1) and (2).

Lemma 3.3 [20] Let $m$ be an integer and $p$ a prime. Then we have:

$$
\varphi_{p^{i}} \simeq \varphi_{p}^{p^{i-1}} \quad \bmod p
$$

Algebraic 83 Geometric Topology, Volume 6 (2006) 
if we suppose that $p \nmid m$, then:

$$
\varphi_{m p^{i}} \simeq \varphi_{m}^{\phi\left(p^{i}\right)} \quad \bmod p
$$

where $\phi$ denotes the Euler $\phi$-function.

Now we consider again the algebra of $q$-divided polynomials $\Gamma_{R}(t, q)$ in the case $R=K(m)$.

Lemma 3.4 The following decompositions hold:

(a) if $p=0$ (see also [22]):

$$
\Gamma_{K(m)}(t, q) \simeq K(m)\left[u_{m}\right] \otimes K(m)\left[u_{1}\right] /\left(u_{1}^{m}\right) ;
$$

(b) if $p \neq 0$ :

$$
\Gamma_{K(p)}(t, q) \simeq \bigotimes_{i=0}^{\infty} K(p)\left[u_{p^{i}}\right] /\left(u_{p^{i}}^{p}\right)
$$

(c) if $p \neq 0$ and $p \nmid m$ :

$$
\Gamma_{K(m)}(t, q) \simeq K(m)\left[u_{1}\right] /\left(u_{1}^{m}\right) \otimes \bigotimes_{i=0}^{\infty} K(m)\left[u_{p^{i} m}\right] /\left(u_{p^{i} m}^{p}\right) ;
$$

with $\operatorname{deg} u_{j}=j$.

Proof The proof of (a) is given in [22].

For (b) the isomorphism is given as follows:

$$
t_{i} \mapsto \frac{u_{1}^{k_{0}} u_{p}^{k_{1}} \cdots u_{p^{r}}^{k_{r}}}{[i] ! / \varphi_{p}^{[i] \varphi_{p}}}
$$

where $k_{r} \cdots k_{0}$ is the expression of $i$ in base $p$ and $x_{\varphi_{p}}$ is the maximal exponent $l$ such that $\varphi_{p}^{l}$ divides $x$.

For (c) we have the isomorphism given by

$$
t_{i} \mapsto \frac{u_{1}^{k} u_{m}^{k_{0}} u_{m p}^{k_{1}} \cdots u_{m p^{r}}^{k_{r}}}{[i] ! / \varphi_{m}^{[i] !_{\varphi m}}}
$$

where $k$ is the remainder of the division of $i$ by $m$ and $k_{r} \cdots k_{0}$ is the expression of $(i-k) / m$ in base $p$. 
The Lemma follows from the next key observation: if $k_{r} \cdots k_{0}$ is the expression of $i$ in the base $p$ and $k_{r}^{\prime} \cdots k_{0}^{\prime}$ is the expression for $j$ (resp. $k, k_{r} \cdots k_{0}$ and $k^{\prime}, k_{r}^{\prime} \cdots k_{0}^{\prime}$ are the numbers associated to $i$ and $j$ as in (9)), then the polynomial $\varphi_{p}$ (resp. $\varphi_{m}$ ) does not divide $\left[\begin{array}{c}i+j \\ i\end{array}\right]$ if and only if the expression for $i+j$ in base $p$ is given by $h_{r} \cdots h_{0}$, with $h_{l}=k_{l}+k_{l}^{\prime}$ for $l=0, \ldots, r$ (resp. the numbers associated to $i+j$ are $h, h_{r} \cdots h_{0}$, with $h=k+k^{\prime}, h_{l}=k_{l}+k_{l}^{\prime}$ for $\left.l=0, \ldots, r\right)$.

The cohomology rings of $R[u]$ and $R[u] /\left(u^{i}\right)$ are already known. In fact we have:

Lemma 3.5 $[22] \quad H^{*}(R[u]) \simeq \Lambda[x], \operatorname{deg}(x)=\operatorname{deg}(u), \operatorname{dim}(x)=1$

$$
H^{*}\left(R[u] /\left(u^{n}\right)\right) \simeq\left\{\begin{array}{cc}
R[x] & \text { for } n=2 \\
R[y] \otimes \Lambda[x] & \text { for } n>2
\end{array}\right.
$$

where $\operatorname{deg}(x)=\operatorname{deg}(u), \operatorname{deg}(y)=n \operatorname{deg}(u), \operatorname{dim}(x)=1, \operatorname{dim}(y)=2$ and $\Lambda[x]$ is the exterior algebra over the ring $R$ in the variable $x$.

We remark that generators of the rings in the Lemma can be given as follows: a representative $x$ is given by the dual class of $u$. Moreover in characteristic $p=0$, a representative of $y$ is given by

$$
\sum_{i=1}^{n-1}\left(\begin{array}{c}
n \\
i
\end{array}\right)\left(u^{i} \otimes u^{n-i}\right)^{*}
$$

and with $p \neq 0$, if $n$ is a power of $p$, we can choose as a representative

$$
\frac{1}{p} \sum_{i=1}^{n-1}\left(\begin{array}{c}
n \\
i
\end{array}\right)\left(u^{i} \otimes u^{n-i}\right)^{*},
$$

where the notation $\left(u^{i} \otimes u^{n-i}\right)^{*}$ means the dual class of $\left(u^{i} \otimes u^{n-i}\right)$.

\section{Computations and results}

Now we can calculate the cohomology of $\Gamma_{K_{p}}(t, q)$ (and so the homology of $\operatorname{Br}(*)$ with coefficients in the local system $K(m)$ ) applying the fact that the cohomology of a tensor product of algebras is the tensor product of the cohomology of the factors.

Applying Lemma 3.5 we have the following straightforward results: 
Theorem 2 [22] If $p=0$ and $m=2$ then

$\begin{aligned} & H_{*}(\operatorname{Br}(*) ; K(2)) \simeq \Lambda\left[x_{2}\right] \otimes K(2)\left[x_{1}\right] ; \\ \text { for } m>2: & H_{*}(\operatorname{Br}(*) ; K(m)) \simeq \Lambda\left[x_{m}\right] \otimes K(m)\left[y_{m}\right] \otimes \Lambda\left[x_{1}\right] ;\end{aligned}$

with $\operatorname{deg} x_{i}=i, \operatorname{dim} x_{i}=i-1, \operatorname{deg} y_{m}=m, \operatorname{dim} y_{m}=m-2$.

Theorem 3 Let $p$ be a prime and $m$ be a positive integer, such that $p \nmid m$. We have the following cases:

(a) if $p=2: \quad H_{*}(\mathrm{Br}(*) ; K(2)) \simeq \bigotimes_{i=0}^{\infty} K(2)\left[x_{2^{i}}\right]$;

$$
H_{*}(\operatorname{Br}(*) ; K(m)) \simeq K(m)\left[y_{m}\right] \otimes \Lambda\left[x_{1}\right] \otimes \bigotimes_{i=0}^{\infty} K(m)\left[x_{m 2^{i}}\right]
$$

(b) if $p>2$ and $m=2$ :

$$
\begin{gathered}
H_{*}(\operatorname{Br}(*) ; K(p)) \simeq \bigotimes_{i=0}^{\infty}\left(K(p)\left[y_{p^{i+1}}\right] \otimes \Lambda\left[x_{p^{i}}\right]\right) ; \\
H_{*}(\operatorname{Br}(*) ; K(2)) \simeq K(2)\left[x_{1}\right] \otimes \bigotimes_{i=0}^{\infty}\left(K(2)\left[y_{2 p^{i+1}}\right] \otimes \Lambda\left[x_{2 p^{i}}\right]\right)
\end{gathered}
$$

(c) if $p>2$ and $m>2$ :

$$
H_{*}(\operatorname{Br}(*) ; K(m)) \simeq K(m)\left[y_{m}\right] \otimes \Lambda\left[x_{1}\right] \bigotimes_{i=0}^{\infty}\left(K(m)\left[y_{p^{i+1} m}\right] \otimes \Lambda\left[x_{p^{i} m}\right]\right) ;
$$

where $\operatorname{deg} x_{i}=\operatorname{deg} y_{i}=i, \operatorname{dim} x_{i}=i-1, \operatorname{dim} y_{i}=i-2$.

We want to use these results to compute the homology of $\operatorname{Br}(*)$ with coefficients in the local system $A$ over the ring $A=K\left[q^{ \pm 1}\right]$ with the same twisting defined as in Section 2 .

The exact sequence

$$
1 \rightarrow \operatorname{Br}(n)^{\prime} \hookrightarrow \operatorname{Br}(n) \rightarrow \mathbb{Z} \rightarrow 1,
$$

tells us that the homology $H_{*}(\operatorname{Br}(n) ; A)$ is $H_{*}\left(\operatorname{Br}(n)^{\prime} ; K\right)$ as an $A$-module (see for example [22], [11] or [7]); since for $n \neq 3,4, \operatorname{Br}(n)^{\prime \prime}=\operatorname{Br}(n)^{\prime}$ (see [18] for a proof of this), we have that $H_{0}\left(\operatorname{Br}(n)^{\prime} ; K\right)=K, H_{1}\left(\operatorname{Br}(n)^{\prime} ; K\right)=0$. Moreover the $A$-action on $H_{0}$ is trivial and so $H_{0}(\operatorname{Br}(n) ; A)=A /(q+1)$ as an $A$-module. Moreover we have: 
Lemma 4.1 [22] The $R$-modules $H_{l}(\operatorname{Br}(n), A)(n>1, l>0)$ are annihilated by multiplication by $[n]$ !.

Let us consider a polynomial $a \in A$. We can consider the set $S_{a}$ of all elements $b \in A$ that are prime with $a$. It is clear that $S_{a}$ is a multiplicatively closed set. We write $A_{(a)}$ for the localization $A_{S_{a}}$ of the ring $A$ respect to the set $S_{a}$.

It follows from Lemma 3.1 that for $p=0, \varphi_{m}$ is invertible in $A_{\left(\varphi_{n}\right)}$ if and only if $m \neq n$; for $p \neq 0, \varphi_{m}$ is invertible in $A_{\left(\varphi_{n}\right)}$ if and only if $n \neq m p^{i}$ and $m \neq n p^{i}$, $\forall i \geq 1$.

The following decompositions hold for the homology of $\operatorname{Br}(n)$ with coefficients in the local system $A$ :

Lemma 4.2 Let $n>1$. For $p=0$ we have:

$$
H_{*}(\operatorname{Br}(n) ; A) \simeq \bigoplus_{m=2}^{\infty} H_{*}\left(\operatorname{Br}(n) ; A_{\left(\varphi_{m}\right)}\right) ;
$$

for $p \neq 0$ :

$$
H_{*}(\operatorname{Br}(n) ; A) \simeq \bigoplus_{p \nmid m \text { or } m=p} H_{*}\left(\operatorname{Br}(n) ; A_{\left(\varphi_{m}\right)}\right) .
$$

Proof Consider the homomorphism

$$
i_{m *}: H_{*}(\operatorname{Br}(n) ; A) \rightarrow H_{*}\left(\operatorname{Br}(n) ; A_{\left(\varphi_{m}\right)}\right)
$$

induced by the injection $i_{m}: A \hookrightarrow A_{\left(\varphi_{m}\right)}$. We extend in a natural way the map $i_{m *}$ through the tensor product with $A_{\left(\varphi_{m}\right)}$ and we get the new map

$$
\widetilde{i_{m}}: H_{*}(\operatorname{Br}(n) ; A) \otimes_{A} A_{\left(\varphi_{m}\right)} \rightarrow H_{*}\left(\operatorname{Br}(n) ; A_{\left(\varphi_{m}\right)}\right) .
$$

Using Lemmas 3.1, 3.3 and 4.1 it is easy to see that in order to prove Lemma 4.2 it is enough to show that the map $\widetilde{i_{m}}$ is an isomorphism.

First we prove the injectivity of $\tilde{i_{m}}$. Let $\alpha$ be a representative of an element $v$ in $H_{*}(\operatorname{Br}(n) ; A) \otimes_{A} A_{\left(\varphi_{m}\right)}$. If the corresponding class of $\widetilde{i_{m}} v$ is zero, and so $\widetilde{i_{m}} \alpha$ is a boundary, then there exists an element $\beta$ such that $d \beta=\widetilde{i_{m}} \alpha$. Multiplying $\beta$ by an appropriate polynomial $\psi$ prime with $\varphi_{m}$, we get an element $\beta^{\prime}=\psi \beta$ that belongs to the resolution of $\operatorname{Br}(n)$ over $A$, so $d \beta^{\prime}=\psi \alpha$. This means that $\psi \alpha$ belongs to the zero class in $H_{*}(\operatorname{Br}(n) ; A)$ and, since $\psi$ is invertible in $A_{\left(\varphi_{m}\right)}, \alpha$ belongs to the zero class in $H_{*}(\operatorname{Br}(n) ; A) \otimes_{A} A_{\left(\varphi_{m}\right)}$. This proves the injectivity of $\widetilde{i_{m}}$.

To prove the surjectivity of $\widetilde{i_{m}}$ we consider a class $w$ in $H_{*}\left(\operatorname{Br}(n) ; A_{\left(\varphi_{m}\right)}\right)$ and we choose a representative $\beta$ for $w$. Multiplying $\beta$ by an appropriate polynomial $\theta$ prime 
with $\varphi_{m}$ we get an element $\beta^{\prime}=\theta \beta$ in the resolution for $H_{*}(\operatorname{Br}(n) ; A)$ and we have that

$$
\widetilde{i_{m}}\left(\beta^{\prime} \otimes \theta^{-1}\right)=\beta
$$

This completes the proof.

The next step is to compute $H_{*}\left(\operatorname{Br}(n) ; A_{\left(\varphi_{m}\right)}\right)$. To do this, consider the following short exact sequence:

$$
0 \rightarrow A_{\left(\varphi_{m}\right)} \stackrel{\varphi_{m}}{\rightarrow} A_{\left(\varphi_{m}\right)} \stackrel{\pi}{\rightarrow} K(m) \rightarrow 0
$$

where the first map is multiplication by $\varphi_{m}$. We want to study the corresponding homology long exact sequence:

$$
\begin{aligned}
& \ldots \quad \stackrel{\pi_{*}}{\rightarrow} H_{l+1}(\mathrm{Br}(*) ; K(m)) \stackrel{\beta}{\rightarrow} \\
& \stackrel{\beta}{\rightarrow} H_{l}\left(\operatorname{Br}(*) ; A_{\left(\varphi_{m}\right)}\right) \stackrel{\left(\varphi_{m}\right)_{*}}{\rightarrow} H_{l}\left(\operatorname{Br}(*) ; A_{\left(\varphi_{m}\right)}\right) \stackrel{\pi_{*}}{\rightarrow} H_{l}(\operatorname{Br}(*) ; K(m)) \stackrel{\beta}{\rightarrow} \\
& \stackrel{\beta}{\rightarrow} H_{l-1}\left(\operatorname{Br}(*) ; A_{\left(\varphi_{m}\right)}\right) \stackrel{\left(\varphi_{m}\right)_{*}}{\rightarrow}
\end{aligned}
$$

We can decompose $H_{l}\left(\operatorname{Br}(*) ; A_{\left(\varphi_{m}\right)}\right)$ as a direct sum of terms $A /\left(\psi^{i}\right)$, where $\psi$ is a prime factor of $\varphi_{m}$. So, if $H_{l}\left(\operatorname{Br}(*) ; A_{\left(\varphi_{m}\right)}\right)$ has a direct summand $A /\left(\psi^{i}\right)$, generated by an element $v$, it follows that $H_{l+1}(\operatorname{Br}(*) ; K(m))$ and $H_{l}(\operatorname{Br}(*) ; K(m))$ have as direct summand a copy of $A /(\psi)$ generated respectively by $w$ and $w^{\prime}$ and we have that

and

$$
\beta w=\psi^{i-1} v
$$

$$
\pi_{*} v=w^{\prime} .
$$

In Theorem 2 (case $p=0$ ) we have these maps (see also [22]):

$$
\beta x_{m}=\widetilde{y_{m}}, \pi_{*} \widetilde{y_{m}}=y_{m}, \beta x_{1}=0 .
$$

while in Theorem 3 (case $p \neq 0$ ), the homomorphisms act as follows:

$$
\begin{gathered}
\beta y_{i}=0, \quad \beta x_{1}=0, \\
\beta x_{2^{i}}=\varphi_{2}^{2^{i-1}-1}{\widetilde{x_{2^{i-1}}}}^{2} ; \quad \beta x_{p^{i}}=\varphi_{p}^{p^{i-1}-1} \widetilde{y_{p^{i}}} \quad \text { for } p>2 ; \\
\beta x_{m p^{i}}=\varphi_{m}^{\phi\left(p^{i}\right)-1} \widetilde{y_{m p^{i}}} \quad \text { for } i>0 \text { or } m>2 ;
\end{gathered}
$$

where $\widetilde{x_{i}}$ and $\widetilde{y_{i}}$ are defined such that:

$$
\pi_{*} \widetilde{x_{i}}=x_{i} ; \pi_{*} \widetilde{y_{i}}=y_{i} .
$$

We can now state the following result:

Algebraic ${ }^{6} \mathcal{G}$ Geometric Topology, Volume 6 (2006) 
Proposition 4.3 For $p=0$ we have, for $m=2$ :

$$
\begin{aligned}
& H_{*}\left(\operatorname{Br}(*) ; A_{\left(\varphi_{2}\right)} \simeq A_{\left(\varphi_{2}\right)}\left[x_{1}\right] /\left(\varphi_{2} x_{1}^{2}\right) ;\right. \\
\text { and for } m>2: & H_{*}\left(\operatorname{Br}(*) ; A_{\left(\varphi_{m}\right)}\right) \simeq A_{\left(\varphi_{m}\right)}\left[x_{1}, y_{m}\right] /\left(x_{1}^{2}, \varphi_{m} y_{m}\right) ;
\end{aligned}
$$

if $p \neq 0$ and $p \nmid m$ we have the following cases:

(a) for $p=2$ :

$$
H_{*}\left(\operatorname{Br}(*) ; A_{\left(\varphi_{2}\right)}\right) \simeq A_{\left(\varphi_{2}\right)}\left[\begin{array}{c}
x_{1}, x_{2^{j}}^{2}, \\
x_{2^{i}}^{2} x_{2^{i}} \cdots x_{2^{i h}}
\end{array}\right] /\left(\varphi_{2}^{2^{i}} x_{2^{i}}^{2}\right)
$$

with $0 \leq i, i+1<i_{1}<\cdots<i_{h}, 0<j$;

$$
H_{*}\left(\operatorname{Br}(*) ; A_{\left(\varphi_{m}\right)}\right) \simeq A_{\left(\varphi_{m}\right)}\left[\begin{array}{c}
x_{1}, y_{m}, x_{m 2^{i}}^{2}, \\
x_{m 2^{i}}^{2} x_{m 2^{i_{1}} \cdots x_{m 2^{i} h}}, \\
y_{m} x_{m 2^{j_{1}}} \cdots x_{m 2^{j_{h}}}
\end{array}\right] /\left(\begin{array}{c}
x_{1}^{2}, \varphi_{m} y_{m}, \\
\varphi_{m}^{2^{i}} x_{m 2^{i}}^{2}
\end{array}\right)
$$

with $0 \leq i, i+1<i_{1}<\cdots<i_{h}, 0<j_{1}<\cdots<j_{h}$;

(b) for $p>2$ and $m=2$ :

$$
H_{*}\left(\operatorname{Br}(*) ; A_{\left(\varphi_{p}\right)}\right) \simeq A_{\left(\varphi_{p}\right)}\left[\begin{array}{c}
x_{1}, y_{p^{i}}, \\
y_{p^{i}} x_{p^{i_{1}}} \cdots x_{p^{i h}}
\end{array}\right] /\left(\begin{array}{c}
x_{p^{i}}^{2} \\
\varphi_{p}^{p^{i}} y_{p^{i}}
\end{array}\right)
$$

with $0<i<i_{1}<\cdots<i_{h}$;

$$
H_{*}\left(\operatorname{Br}(*) ; A_{\left(\varphi_{2}\right)}\right) \simeq A_{\left(\varphi_{2}\right)}\left[\begin{array}{c}
x_{1}, y_{2 p^{i}}, \\
x_{1}^{2} x_{2 p^{j_{1}} \cdots x_{2 p^{j_{h}}}} \\
y_{2 p^{i} x_{2 p^{i}} \cdots x_{2 p^{i}}}
\end{array}\right] /\left(\begin{array}{c}
\varphi_{2} x_{1}^{2}, x_{2 p^{i}}^{2}, \\
\varphi_{2}^{\phi\left(p^{i}\right)} y_{2 p^{i}}
\end{array}\right)
$$

with $0<i<i_{1}<\cdots<i_{h}, 0<j_{1}<\cdots<j_{h}$;

(c) for $p>2$ and $m>2$ :

$$
H_{*}\left(\operatorname{Br}(*) ; A_{\left(\varphi_{m}\right)}\right) \simeq A_{\left(\varphi_{m}\right)}\left[\begin{array}{c}
x_{1}, y_{m p^{i}}, \\
y_{m p^{i}} x_{m p^{i_{1}}} \cdots x_{m p^{i h}}
\end{array}\right] /\left(\begin{array}{c}
x_{1}^{2}, x_{m p^{i}}^{2}, \\
\varphi_{m}^{\phi\left(p^{i}\right)} y_{m p^{i}}
\end{array}\right) ;
$$

with $0 \leq i<i_{1}<\cdots<i_{h}$;

in all cases $\operatorname{deg} x_{i}=\operatorname{deg} y_{i}=i, \operatorname{dim} x_{i}=i-1, \operatorname{dim} y_{i}=i-2$.

In order to get $H_{*}\left(\operatorname{Br}(n) ; \mathbb{Z}\left[q^{ \pm 1}\right]\right)$ we still have to compute the Bockstein homomorphism $\beta_{p}$ associated to the short exact sequence

$$
0 \rightarrow \mathbb{Z}_{p} \hookrightarrow \mathbb{Z}_{p^{2}} \rightarrow \mathbb{Z}_{p} \rightarrow 0
$$


We'll see that, as in the case of trivial coefficients (see [12] or [24]), there is no $p^{2}$-torsion in the homology of braid groups.

In case (a) of Proposition 4.3 the Bockstein acts as follows (the coefficients $\psi_{i, j}$ are those defined in Corollary 3.2):

$$
\begin{aligned}
& \beta_{2} x_{1}=0, \quad \beta_{2} x_{2^{i}}^{2}=0, \\
& \beta_{2} x_{2^{i}}^{2} x_{2^{i_{1}}} \cdots x_{2^{i_{h}}}=\sum_{s=1}^{h}\left(\psi_{2^{i_{s}}, 2^{i}} x_{2^{i}}^{2} x_{2^{i_{s}-1}}^{2} \prod_{t \neq s} x_{2^{i_{t}}}\right) \\
& \beta_{2} y_{m}=0, \beta_{2} x_{m 2^{i}}^{2}=0 \text {, } \\
& \beta_{2} x_{m 2^{i}}^{2} x_{m 2^{i^{i}}} \cdots x_{m 2^{i h}}=\sum_{s=1}^{h}\left(\psi_{m 2^{i_{s}-1}, m 2^{i}} x_{m 2^{i}}^{2} x_{m 2^{i_{s}-1}}^{2} \prod_{t \neq s} x_{m 2^{i_{t}}}\right) \text {, } \\
& \beta_{2} y_{m} x_{m 2^{i_{1}}} \cdots x_{m 2^{i_{h}}}=\sum_{s=1}^{h}\left(\psi_{m 2^{i_{s}-1}, m} y_{m} x_{m 2^{i_{s}-1}}^{2} \prod_{s \neq t} x_{m 2^{i_{t}}}\right) \text {; }
\end{aligned}
$$

in case (b) we have:

$$
\begin{aligned}
& \beta_{p} x_{1}=0, \beta_{p} y_{p^{i}}=0, \\
& \beta_{p} y_{p^{i}} x_{p^{i_{1}}} \cdots x_{p^{i_{h}}}=-\sum_{s=1}^{h}\left(\psi_{p^{i_{s}}, p^{i}} y_{p^{i}} y_{p^{i_{s}-1}} \prod_{s \neq t} x_{p^{i_{t}}}\right) \text {; } \\
& \beta_{p} x_{1}^{2} x_{2 p^{i_{1}}} \cdots x_{2 p^{i_{h}}}=-\sum_{s=1}^{h}\left(\psi_{2 p^{i_{s}, 2 p^{i}}} x_{1}^{2} y_{2 p^{i_{s}-1}} \prod_{s \neq t} x_{2 p^{i_{t}}}\right) ; \\
& \beta_{p} y_{2 p^{i}}=0 \text {, }
\end{aligned}
$$

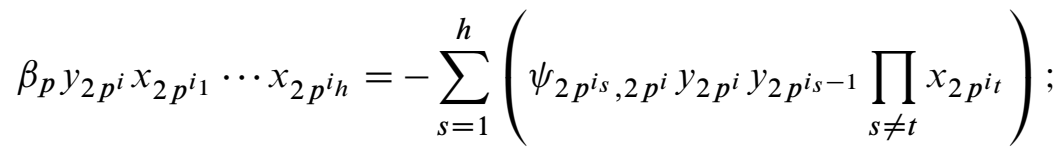

finally in case (c) the map is:

$$
\begin{gathered}
\beta_{p} x_{1}=0, \beta_{p} y_{m p^{i}}=0 \\
\beta_{p} y_{m p^{i}} x_{m p^{i_{1}}} \cdots x_{m p^{i_{h}}}=-\sum_{s=1}^{h}\left(\psi_{m p^{i_{s}}, m p^{i}} y_{m p^{i}} y_{m p^{i_{s}-1}} \prod_{s \neq t} x_{m p^{i_{t}}}\right) .
\end{gathered}
$$

Lemma 4.4 The homology groups $H_{*}\left(\operatorname{Br}(*) ; \mathbb{Z}\left[q^{ \pm 1}\right]\right)$ have no $p^{2}$-torsion. 
Proof Notice that the monomials $1, x_{1}$ generate the groups $H_{0}\left(\operatorname{Br}(0), \mathbb{Z}\left[q^{ \pm 1}\right]\right)$ and $H_{0}\left(\operatorname{Br}(1), \mathbb{Z}\left[q^{ \pm 1}\right]\right)$ and that both these modules are equal to $\mathbb{Z}\left[q^{ \pm 1}\right]$. For $i \geq 2$ the groups $H_{0}\left(\operatorname{Br}(i), \mathbb{Z}\left[q^{ \pm 1}\right]\right)=\mathbb{Z}$ are generated by the monomials $x_{1}^{i}$. Now consider the following monomials:

$$
\begin{aligned}
& y_{m}^{i}, \quad y_{m}^{i} x_{1} \quad(\text { case } p=0) \\
& \left.\left.\begin{array}{lc}
x_{2^{j}}^{2 i}, & x_{2^{j}}^{2 i} x_{1}, \\
x_{m 2^{j}}^{2 i}, & x_{m 2^{j}}^{2 i} x_{1}
\end{array}\right\} \quad \text { (case } p=2\right) \\
& y_{p^{j}}^{i}, \quad y_{p^{j}}^{i} x_{1}, \\
& \left.y_{2 p^{j}}^{i}, \quad y_{2 p^{j}}^{i} x_{1}, \quad \text { (case } p>2\right) \\
& y_{m p^{j}}^{i}, y_{m p^{j}}^{i} x_{1}
\end{aligned}
$$

Because of the computations over $\mathbb{Q}\left[q^{ \pm 1}\right]$ ([14], [22]), their liftings generate a free $\mathbb{Z}$-module of type $\mathbb{Z}\left[q^{ \pm 1}\right] /\left(\varphi_{h}\right)$ in dimension $d$ in the homology of $\operatorname{Br}(n)$ whenever $n=k h$ or $n=k h+1$ and $d=k(h-2)$ and the Bockstein is zero for all these monomials.

All the other monomials lift to torsion classes and all these classes don't have $p^{2}-$ torsion for any prime $p$. To prove this it is enough to show that in the submodule $M_{p} \subset H_{*}\left(\operatorname{Br}(*), \mathbb{Z}_{p}\left[q^{ \pm 1}\right]\right)$ generated by all the monomials different from the ones in (12) or (13), we have that

$$
\operatorname{ker} \beta_{p}=\operatorname{im} \beta_{p} .
$$

Let us consider the set $S$ of the monomials that appear in the polynomial rings of parts (a), (b) and (c) of Proposition 4.3 and different from these in (12) and (13).

Let us say that a monomial $w$ rises up to a monomial $w^{\prime}$ is $w$ appears as a summand in $\beta_{p} w^{\prime}$. We call $w$ a basic monomial if it doesn't appear as a summand in $\beta_{p} w^{\prime}$ for any monomial $w^{\prime}$. We also say that a monomial $w$ is a child of $w^{\prime}$ if $w^{\prime}$ is basic and we can rise up from $w$ to $w^{\prime}$ in a finite number of steps. We notice that in general a basic polynomial can be of the following form:

$$
w=x_{i_{1}}^{2 a_{1}} \cdots x_{i_{k}}^{2 a_{k}} y_{j_{1}}^{b_{1}} \cdots y_{j_{h}}^{b_{k}} x_{l_{1}} \cdots x_{l_{s}} .
$$

Let $\Delta_{w}$ be the set of all monomials that are children of $w$ (including $w$ itself). It is easy to see that $\Delta_{w}$ is in bijection with the set of the parts of $\{1, \ldots, s\}$ (with $s \geq 1$ ) if $l_{1}, \ldots, l_{s}$ are all different from 1 , or with the set of the parts of $\{1, \ldots, s-1\}$ (with $s \geq 2$ ) if one of $l_{1}, \ldots, l_{s}$ is 1 .

Let we say that a monomial $w$ has $\varphi$-torsion (over the ring $\mathbb{Z}_{p}\left[q^{ \pm 1}\right]$ ) if it generates a module isomorphic to $\mathbb{Z}_{p}\left[q^{ \pm 1}\right] /(\varphi)$. If a monomial $w$ has $\varphi$-torsion over $\mathbb{Z}_{p}\left[q^{ \pm 1}\right]$ 
then all the other monomials children of $w$ have the same torsion. Moreover consider the algebraic complex $\left(M_{w}, \beta_{p}\right)$ given by the module $M_{w}$ generated (over $\mathbb{Z}_{p}\left[q^{ \pm 1}\right]$ ) by all the monomials in $\Delta_{w}$ and with the restriction of $\beta_{p}$ to $M_{w}$ as a boundary map: we have that $\left(M_{w}, \beta_{p}\right)$ is isomorphic to the algebraic complex that computes the reduced homology of the $(s-1)$-dimensional simplex with constant coefficients, over the $\operatorname{ring} \mathbb{Z}_{p}\left[q^{ \pm 1}\right] /(\varphi)$ and so $\operatorname{ker} \beta_{p}=\operatorname{im} \beta_{p}$ on $M_{w}$.

One can check that for every monomial $w$ in $S$ there exists one and only one basic monomial $w^{\prime}$ such that $w$ is a child of $w^{\prime}$. This implies that the family of all different sets $\Delta_{w}$ gives a partition of $S$ and so $\operatorname{ker} \beta_{p}=\operatorname{im} \beta_{p}$ on all the module $M$. The Lemma follows.

\section{Main Result}

As a consequence of the last Lemma and of the previous computations, we can now state our main Theorem. Recall that the ring $H_{*}\left(\operatorname{Br}(*) ; R\left[q^{ \pm 1}\right]\right)$ is the bigraded direct sum of the modules $H_{i}\left(\operatorname{Br}(n) ; R\left[q^{ \pm 1}\right]\right)=H_{i}\left(\mathbf{F}_{1}(n), R\right)$, where $\mathbf{F}_{1}(n)$ is the Milnor fiber of the discriminant singularity for $\operatorname{Br}(n)$.

Theorem 4 Set $\operatorname{deg} x_{i}=\operatorname{deg} y_{i}=i, \operatorname{dim} x_{1}=0, \operatorname{dim} x_{i}=i-1, \operatorname{dim} y_{i}=i-2$. Then:

$$
\begin{gathered}
H_{*}\left(\operatorname{Br}(*) ; \mathbb{Q}\left[q^{ \pm 1}\right]\right) \simeq \mathbb{Q}\left[q^{ \pm 1}\right]\left[x_{1}, y_{m}, m>2\right] /\left(\varphi_{2} x_{1}^{2}, \varphi_{m} y_{m}\right) \\
H_{*}\left(\operatorname{Br}(*) ; \mathbb{Z}_{2}\left[q^{ \pm 1}\right]\right) \simeq \mathbb{Z}_{2}\left[q^{ \pm 1}\right]\left[\begin{array}{c}
x_{1}, y_{m}, x_{2^{i+1}}^{2}, x_{m 2^{i}}^{2}, \\
x_{2^{i}}^{2} x_{2^{i_{1}}} \cdots x_{2^{i} h}, \\
x_{m 2^{i}}^{x_{m 2^{i}} \cdots x_{m 2^{i} h},} \\
y_{m} x_{m 2^{j_{1}} \cdots x_{m 2^{j}},}, \\
m \geq 2,2 \nmid m
\end{array}\right] /\left(\begin{array}{c}
\varphi_{2}^{2^{i}} x_{2^{i}}^{2}, \\
\varphi_{m} y_{m}, \\
\varphi_{m}^{2^{i}} x_{m 2^{i}}^{2}
\end{array}\right)
\end{gathered}
$$

with $0 \leq i, i+1<i_{1}<\cdots<i_{h}, 0<j_{1}<\cdots<j_{h}$;

for $p>2$ :

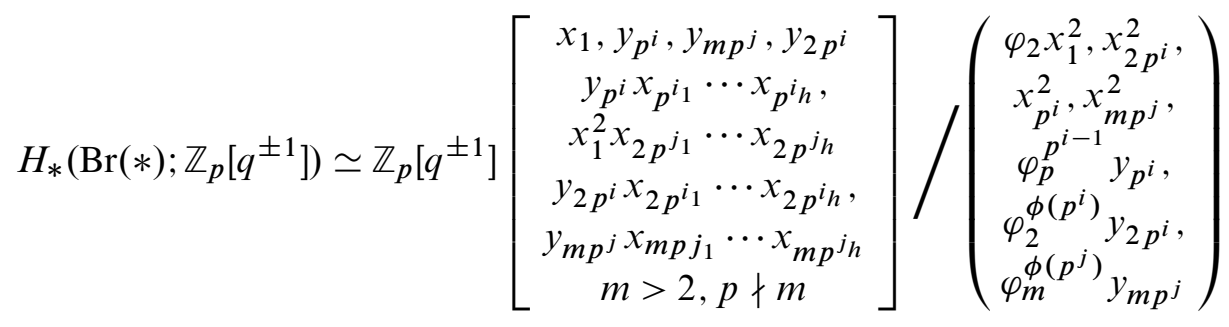

Algebraic 83 Geometric Topology, Volume 6 (2006) 
with $0<i<i_{1}<\cdots<i_{h}, 0 \leq j<j_{1}<\cdots<j_{h}$. Finally, using the notation of the proof of Lemma 4.4, we have:

$$
\begin{gathered}
H_{*}\left(\operatorname{Br}(*) ; \mathbb{Z}\left[q^{ \pm 1}\right]\right) \simeq \\
\mathbb{Z}\left[q^{ \pm 1}\right]\left[x_{1}, y_{m}, m>2\right] /\left(\varphi_{2} x_{1}^{2}, \varphi_{m} y_{m}\right) \oplus \bigoplus_{p \geq 2} \beta_{p} M_{p} .
\end{gathered}
$$

In tables 1, 2, 3 and 4 we give the explicit computations for some cases. The results in Table 3 correspond to that in [14] for cohomology. We use the notation $\varphi_{h}^{i}$ for the module $\mathbb{Z}_{p}[q] /\left(\varphi_{h}^{i}\right)$ or $\mathbb{Q}[q] /\left(\varphi_{h}^{i}\right)$ (note that $R\left[q^{ \pm 1}\right] /\left(\varphi_{h}^{i}\right)=R[q] /\left(\varphi_{h}^{i}\right)$ ). In Table 4 we describe the additive structure of the integral homology of the fiber $\mathbf{F}_{1}(n)$.

\begin{tabular}{|c|c|c|c|c|c|c|c|c|c|}
\hline$n$ & 2 & 3 & 4 & 5 & 6 & 7 & 8 & 9 & 10 \\
\hline$H_{0}$ & $\varphi_{2}$ & $\varphi_{2}$ & $\varphi_{2}$ & $\varphi_{2}$ & $\varphi_{2}$ & $\varphi_{2}$ & $\varphi_{2}$ & $\varphi_{2}$ & $\varphi_{2}$ \\
\hline$H_{1}$ & & $\varphi_{3}$ & $\varphi_{3}$ & & & & & & \\
\hline$H_{2}$ & & & $\varphi_{2}^{2}$ & $\varphi_{2}^{2}$ & $\varphi_{2} \oplus \varphi_{3}$ & $\varphi_{2} \oplus \varphi_{3}$ & $\varphi_{2}$ & $\varphi_{2}$ & $\varphi_{2}$ \\
\hline$H_{3}$ & & & & $\varphi_{5}$ & $\varphi_{2} \oplus \varphi_{5}$ & $\varphi_{2}$ & $\varphi_{2}$ & $\varphi_{2} \oplus \varphi_{3}$ & $\varphi_{2} \oplus \varphi_{3}$ \\
\hline$H_{4}$ & & & & $\varphi_{3}$ & $\varphi_{3}$ & $\varphi_{2}^{2}$ & $\varphi_{2}^{2}$ & $\varphi_{2}$ \\
\hline$H_{5}$ & & & & & $\varphi_{7}$ & $\varphi_{7}$ & $\varphi_{3}$ & $\varphi_{2} \oplus \varphi_{3}$ \\
\hline$H_{6}$ & & & & & & & $\varphi_{2}^{4}$ & $\varphi_{2}^{4} \oplus \varphi_{3}$ & $\varphi_{2} \oplus \varphi_{3} \oplus \varphi_{5}$ \\
\hline$H_{7}$ & & & & & & & & $\varphi_{9}$ & $\varphi_{2} \oplus \varphi_{9}$ \\
\hline$H_{8}$ & & & & & & & & & $\varphi_{5}$ \\
\hline
\end{tabular}

Table 1: $H_{*}\left(\operatorname{Br}(n) ; \mathbb{Z}_{2}\left[q^{ \pm 1}\right]\right)$

Now consider the natural embeddings $j_{n}$ : $\operatorname{Br}(n) \hookrightarrow \operatorname{Br}(n+1)$. By definition the direct limit $\lim _{n} \operatorname{Br}(n)$ is the braid group on infinitely many strands $\operatorname{Br}(\infty)$. Notice that the first $p$-torsion class in the groups $H_{*}\left(\operatorname{Br}(n) ; \mathbb{Z}\left[q^{ \pm 1}\right]\right)$ appears for $n=2 p+2$ in dimension $2 p-2$ and is stable; the corresponding generator is $x_{1}^{2} x_{2}^{2}$ for $p=2$ or $x_{1}^{2} y_{2 p}$ for $p>2$. An equivalent result for the cohomology was proven in [8].

As a consequence of Theorem 4, we can compute the stable homology of braid groups, that is, the homology of $\operatorname{Br}(\infty)$ (see also [13]).

Corollary 5.1 We have that $H_{*}\left(\operatorname{Br}(\infty) ; \mathbb{Q}\left[q^{ \pm 1}\right]\right)=\mathbb{Q}$, concentrated in dimension 0 ;

$$
H_{*}\left(\operatorname{Br}(\infty) ; \mathbb{Z}_{2}\left[q^{ \pm 1}\right]\right)=\mathbb{Z}_{2}\left[x_{2}^{2}, x_{2^{i}}, i>1\right]
$$




\begin{tabular}{|c|c|c|c|c|c|c|c|c|c|}
\hline$n$ & 2 & 3 & 4 & 5 & 6 & 7 & 8 & 9 & 10 \\
\hline$H_{0}$ & $\varphi_{2}$ & $\varphi_{2}$ & $\varphi_{2}$ & $\varphi_{2}$ & $\varphi_{2}$ & $\varphi_{2}$ & $\varphi_{2}$ & $\varphi_{2}$ & $\varphi_{2}$ \\
\hline$H_{1}$ & & $\varphi_{3}$ & $\varphi_{3}$ & & & & & & \\
\hline$H_{2}$ & & & $\varphi_{4}$ & $\varphi_{4}$ & $\varphi_{3}$ & $\varphi_{3}$ & & & \\
\hline$H_{3}$ & & & $\varphi_{5}$ & $\varphi_{5}$ & & & $\varphi_{3}$ & $\varphi_{3}$ \\
\hline$H_{4}$ & & & & & $\varphi_{2}^{2}$ & $\varphi_{2}^{2}$ & $\varphi_{2} \oplus \varphi_{4}$ & $\varphi_{2} \oplus \varphi_{4}$ & $\varphi_{2}$ \\
\hline$H_{5}$ & & & & & & $\varphi_{7}$ & $\varphi_{2} \oplus \varphi_{7}$ & $\varphi_{2}$ & $\varphi_{2}$ \\
\hline$H_{6}$ & & & & & & & $\varphi_{8}$ & $\varphi_{8}$ & $\varphi_{5}$ \\
\hline$H_{7}$ & & & & & & & & $\varphi_{3}^{2}$ & $\varphi_{3}^{2}$ \\
\hline$H_{8}$ & & & & & & & & & $\varphi_{10}$ \\
\hline
\end{tabular}

Table 2: $H_{*}\left(\operatorname{Br}(n) ; \mathbb{Z}_{3}\left[q^{ \pm 1}\right]\right)$

\begin{tabular}{|c|c|c|c|c|c|c|c|c|c|}
\hline$n$ & 2 & 3 & 4 & 5 & 6 & 7 & 8 & 9 & 10 \\
\hline$H_{0}$ & $\varphi_{2}$ & $\varphi_{2}$ & $\varphi_{2}$ & $\varphi_{2}$ & $\varphi_{2}$ & $\varphi_{2}$ & $\varphi_{2}$ & $\varphi_{2}$ & $\varphi_{2}$ \\
\hline$H_{1}$ & & $\varphi_{3}$ & $\varphi_{3}$ & & & & & & \\
\hline$H_{2}$ & & & $\varphi_{4}$ & $\varphi_{4}$ & $\varphi_{3}$ & $\varphi_{3}$ & & & \\
\hline$H_{3}$ & & & & $\varphi_{5}$ & $\varphi_{5}$ & & & $\varphi_{3}$ & $\varphi_{3}$ \\
\hline$H_{4}$ & & & & & $\varphi_{6}$ & $\varphi_{6}$ & $\varphi_{4}$ & $\varphi_{4}$ & \\
\hline$H_{5}$ & & & & & & $\varphi_{7}$ & $\varphi_{7}$ & & \\
\hline$H_{6}$ & & & & & & & $\varphi_{8}$ & $\varphi_{8}$ & $\varphi_{5}$ \\
\hline$H_{7}$ & & & & & & & & $\varphi_{9}$ & $\varphi_{9}$ \\
\hline$H_{8}$ & & & & & & & & & $\varphi_{10}$ \\
\hline
\end{tabular}

Table 3: $H_{*}\left(\operatorname{Br}(n) ; \mathbb{Q}\left[q^{ \pm 1}\right]\right)$

and for a prime $p>2$

$$
H_{*}\left(\operatorname{Br}(\infty) ; \mathbb{Z}_{p}\left[q^{ \pm 1}\right]\right)=\mathbb{Z}_{p}\left[y_{2 p^{i}}, x_{2 p^{i}}, i>0\right] /\left(x_{2 p^{i}}^{2}\right)
$$

with $\operatorname{dim} x_{i}=i-1, \operatorname{dim} y_{i}=i-2$. The Bockstein operator acts as follows:

$$
\beta_{2} x_{2^{i}}=x_{2^{i-1}}^{2} ; \quad \beta_{p} y_{i}=0 ; \quad \beta_{p} x_{i}=y_{i} \quad(\text { for } p>2) .
$$

Algebraic 83 Geometric Topology, Volume 6 (2006) 


\begin{tabular}{|c|c|c|c|c|c|c|c|c|c|}
\hline$n$ & 2 & 3 & 4 & 5 & 6 & 7 & 8 & 9 & 10 \\
\hline$H_{0}$ & $\mathbb{Z}$ & $\mathbb{Z}$ & $\mathbb{Z}$ & $\mathbb{Z}$ & $\mathbb{Z}$ & $\mathbb{Z}$ & $\mathbb{Z}$ & $\mathbb{Z}$ & $\mathbb{Z}$ \\
\hline$H_{1}$ & & $\mathbb{Z}^{2}$ & $\mathbb{Z}^{2}$ & & & & & & \\
\hline$H_{2}$ & & & $\mathbb{Z}^{2}$ & $\mathbb{Z}^{2}$ & $\mathbb{Z}_{2} \oplus \mathbb{Z}^{2}$ & $\mathbb{Z}_{2} \oplus \mathbb{Z}^{2}$ & $\mathbb{Z}_{2}$ & $\mathbb{Z}_{2}$ & $\mathbb{Z}_{2}$ \\
\hline$H_{3}$ & & & $\mathbb{Z}^{4}$ & $\mathbb{Z}^{4}$ & & & $\mathbb{Z}^{2}$ & $\mathbb{Z}^{2}$ \\
\hline$H_{4}$ & & & & $\mathbb{Z}^{2}$ & $\mathbb{Z}^{2}$ & $\mathbb{Z}_{3} \oplus \mathbb{Z}^{2}$ & $\mathbb{Z}_{3} \oplus \mathbb{Z}^{2}$ & $\mathbb{Z}_{2} \oplus \mathbb{Z}_{3}$ \\
\hline$H_{5}$ & & & & & $\mathbb{Z}^{6}$ & $\mathbb{Z}^{6}$ & $\mathbb{Z}_{2}^{2}$ & $\mathbb{Z}_{2}^{2}$ \\
\hline$H_{6}$ & & & & & & $\mathbb{Z}^{4}$ & $\mathbb{Z}^{4}$ & $\mathbb{Z}_{2} \oplus \mathbb{Z}^{4}$ \\
\hline$H_{7}$ & & & & & & & $\mathbb{Z}^{6}$ & $\mathbb{Z}^{6}$ \\
\hline$H_{8}$ & & & & & & & & $\mathbb{Z}^{4}$ \\
\hline
\end{tabular}

Table $4: H_{*}\left(\mathbf{F}_{1}(n) ; \mathbb{Z}\right)$

The homology $H_{*}\left(\operatorname{Br}(\infty) ; \mathbb{Z}\left[q^{ \pm 1}\right]\right)$ has no $p^{2}$-torsion for any prime $p$. A presentation of $H_{*}\left(\operatorname{Br}(\infty) ; \mathbb{Z}\left[q^{ \pm 1}\right]\right)$ is given by

$$
\mathbb{Z}\left[\begin{array}{c}
y_{2 p^{i}}, x_{2^{j}}^{2}, \\
x_{2^{i}}^{2} x_{2^{i_{1}}} \cdots x_{2^{i_{h}}} \\
y_{2 p^{j}} x_{2 p^{j_{1}}} \cdots x_{2 p^{j_{h}}}
\end{array}\right] /\left(2 x_{2^{i}}, p y_{2 p^{j}}, x_{2 p^{j}}^{2}\right)
$$

with indices running as follows: $0<i, i+1<i_{1}<\cdots<i_{h}, 0<j<j_{1}<\cdots<j_{h}$ and $p$ in the set of odd primes. The structure of $\mathbb{Z}\left[q^{ \pm 1}\right]$-module is trivial and so the action of $q$ corresponds to multiplication by -1 .

\section{Appendix}

Here we give the proof of a technical lemma stated in Section 3.

Lemma 3.1 Let $m<n$ be two positive integers. Also let $p$ be a prime. Then we have:

$$
\left(\varphi_{m}, \varphi_{n}\right)=\left\{\begin{array}{cl}
\left(\varphi_{m}, p\right) & \text { if } n=m p^{i}, i \geq 1 \\
(1) & \text { otherwise. }
\end{array}\right.
$$

Proof First of all, notice that the polynomials $\varphi_{m}$ are irreducible for all $m \in \mathbb{N}$; hence, the quotient rings $\mathbb{Z}[q] /\left(\varphi_{m}\right)$ are integral domains.

(i) First suppose that $m \nmid n$ and let $l=\operatorname{lcm}(m, n)$. Moreover we set $m^{\prime}=\frac{l}{m}, n^{\prime}=\frac{l}{n}$. We have that $\varphi_{n} \mid \frac{[l]}{[m]}$ and $\varphi_{m} \mid \frac{[l]}{[n]}$. Furthermore $\frac{[l]}{[m]} \cong m^{\prime}\left(\bmod \varphi_{m}\right)$ and $\frac{[l]}{[n]} \cong n^{\prime}$ 
$\left(\bmod \varphi_{n}\right)$. Since we have $\left(m^{\prime}, n^{\prime}\right)=(1)$ it follows that $\left(\varphi_{m}, \varphi_{n}\right)=(1)$. Hence the polynomial $\varphi_{n}$ is invertible in $\mathbb{Z}[q] /\left(\varphi_{m}\right)$ (and $\varphi_{m}$ is invertible in $\mathbb{Z}[q] /\left(\varphi_{n}\right)$ ).

(ii) Now we suppose that $m \mid n$. For a fixed $m$ we want to prove by induction on $n$ that, modulo the multiplication by an invertible element in $\mathbb{Z}[q] /\left(\varphi_{m}\right)$, the following holds:

$$
\begin{aligned}
& \varphi_{n} \cong p \text { if } n=m p^{i}, i \geq 1 \\
& \varphi_{n} \cong 1 \quad \text { otherwise }
\end{aligned}
$$

If $n=m p$ we have that

$$
\frac{[n]}{[m]} \cong p \quad\left(\bmod \varphi_{m}\right)
$$

and so we can write

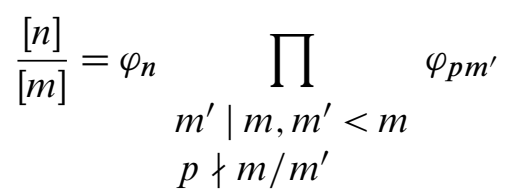

Since all the factors in the product are invertible, it follows that, modulo multiplication by invertible elements in $\mathbb{Z}[q] /\left(\varphi_{m}\right)$, we get $\varphi_{n} \cong p$.

If $n=m p^{i}$, in a similar way the next equality holds:

$$
\frac{[n]}{[m]}=\varphi_{n} \prod_{\substack{1 \leq j<i \\ m^{\prime} \mid m, m^{\prime} p^{j} \neq n \\ p \nmid m / m^{\prime}}} \varphi_{m^{\prime} p^{j}} \cong p^{i} \quad\left(\bmod \varphi_{m}\right)
$$

In the product there are exactly $i-1$ factors congruent to $p$ and all the others are invertible, so modulo invertible elements we have that $\varphi_{n} \cong p$.

Finally we consider the case $n=m p_{1}^{i_{1}} \cdots p_{k}^{i_{k}}$. Let us define the set

$$
\mathcal{I}=\left\{\begin{array}{l|c}
\left(m^{\prime}, j_{1}, \ldots, j_{k}\right) \in \mathbb{N}^{k+1} & m^{\prime} \mid m, \\
0 \leq j_{s} \leq i_{s} \forall s \\
\left(j_{1}, \ldots, j_{k}\right) \neq(0, \ldots, 0), \\
m^{\prime} \neq m \text { if } j_{s}=i_{s} \forall s \\
p_{s} \nmid\left(m / m^{\prime}\right) \quad \forall s \text { s.t. } j_{s} \neq 0
\end{array}\right\}
$$

We have that:

$$
\frac{[n]}{[m]}=\prod_{n^{\prime} \mid n, n^{\prime} \nmid m} \varphi_{n^{\prime}}=\prod_{I \in \mathcal{I}} \varphi_{m^{\prime} p_{1}^{j_{1}} \ldots p_{k}^{j_{k}}} \cong p_{1}^{i_{1}} \cdots p_{k}^{i_{k}} \quad\left(\bmod \varphi_{m}\right)
$$

Algebraic $8 \mathcal{G}$ Geometric Topology, Volume 6 (2006) 
and, by the inductive hypothesis, in the product, for all $s$ there are exactly $i_{s}$ factors congruent to $p_{s}$ and all the other factors are invertible; hence $\varphi_{n}$ must be invertible, too. So the Lemma is proved.

Acknowledgments The author is grateful to M Salvetti for fruitful discussions. The author is also thankful to F R Cohen and to the referee for many useful suggestions.

\section{References}

[1] V I Arnol'd, Braids of algebraic functions and cohomologies of swallowtails, Uspehi Mat. Nauk 23 (1968) 247-248 MR0231828

[2] V I Arnol'd, The cohomology ring of the group of dyed braids, Mat. Zametki 5 (1969) 227-231 MR0242196

[3] E Artin, Theorie der Zöpfe, Abh. Math. Sem. Univ. Hambutg 4 (1925) 42-72

[4] J S Birman, Braids, links, and mapping class groups, Annals of Mathematics Studies 82, Princeton University Press (1974) MR0375281

[5] N Bourbaki, Éléments de mathématique: Groupes et algèbres de Lie. Chapitres 4, 5 et 6, Masson, Paris (1981) MR647314

[6] E Brieskorn, K Saito, Artin-Gruppen und Coxeter-Gruppen, Invent. Math. 17 (1972) 245-271 MR0323910

[7] F Callegaro, On the cohomology of Artin groups in local systems and the associated Milnor fiber, J. Pure Appl. Algebra 197 (2005) 323-332 MR2123992

[8] F Callegaro, Proprietá intere della coomologia dei gruppi di Artin e della fibra di Milnor associata, Master's thesis, Dipartimento di Matematica Univ. di Pisa (June 2003)

[9] F Callegaro, M Salvetti, Integral cohomology of the Milnor fibre of the discriminant bundle associated with a finite Coxeter group, C. R. Math. Acad. Sci. Paris 339 (2004) 573-578 MR2111354

[10] H Cartan, S Eilenberg, Homological algebra, Princeton University Press, Princeton, NJ (1956) MR0077480

[11] D C Cohen, A I Suciu, Homology of iterated semidirect products of free groups, J. Pure Appl. Algebra 126 (1998) 87-120 MR1600518

[12] F R Cohen, The homology of $C_{n+1}$-spaces, $n \geq 0$, from: "The homology of iterated loop spaces", Lecture Notes in Mathematics 533, Springer, Berlin (1976) 207-353 MR0436146

[13] F R Cohen, J Pakianathan, The stable braid group and the determinant of the Burau representation, Cont. Math. to appear arXiv:math.AT/0509577

[14] C De Concini, C Procesi, M Salvetti, Arithmetic properties of the cohomology of braid groups, Topology 40 (2001) 739-751 MR1851561 
[15] C De Concini, C Procesi, M Salvetti, F Stumbo, Arithmetic properties of the cohomology of Artin groups, Ann. Scuola Norm. Sup. Pisa Cl. Sci. (4) 28 (1999) 695-717 MR1760537

[16] È V Frenkel', Cohomology of the commutator subgroup of the braid group, Funktsional. Anal. i Prilozhen. 22 (1988) 91-92 (Russian) MR961774 English translation: Func. Anal. Appl. 22 (1988) 248-250

[17] D B Fuks, Cohomology of the braid group mod 2, Funkcional. Anal. i Priložen. 4 (1970) 62-73 (Russian) MR0274463 English translation: Func. Anal. Appl. 22 (1970) $143-151$

[18] E A Gorin, V J Lin, Algebraic equations with continuous coefficients, and certain questions of the algebraic theory of braids, Mat. Sb. (N.S.) 78 (120) (1969) 579-610 MR0251712

[19] V V Gorjunov, Cohomology of braid groups of series $C$ and D, Trudy Moskov. Mat. Obshch. 42 (1981) 234-242 MR622003

[20] W J Guerrier, The factorization of the cyclotomic polynomials mod $p$, Amer. Math. Monthly 75 (1968) 46 MR0225747

[21] C Landi, Cohomology rings of Artin groups, Atti Accad. Naz. Lincei Cl. Sci. Fis. Mat. Natur. Rend. Lincei (9) Mat. Appl. 11 (2000) 41-65 MR1797053

[22] N S Markaryan, Homology of braid groups with nontrivial coefficients, Mat. Zametki 59 (1996) 846-854, 960 (Russian) MR1445470 English translation: Math. Notes, 59 (1996) 611-617

[23] M Salvetti, The homotopy type of Artin groups, Math. Res. Lett. 1 (1994) 565-577 MR1295551

[24] F V Vaĭnšteĭn, The cohomology of braid groups, Funktsional. Anal. i Prilozhen. 12 (1978) 72-73 (Russian) MR498903 English translation: Func. Anal. Appl. 12 (1978) 135-137

Scuola Normale Superiore, Piazza dei Cavalieri, 7

56126 Pisa, Italy

f.callegaro@sns.it

Received: 30 November 2005 Revised: 22 September 2006 